PROCEEDINGS OF THE

AMERICAN MATHEMATICAL SOCIETY

Volume 134, Number 6, Pages 1631-1634

S 0002-9939(05)08283-3

Article electronically published on December 15, 2005

\title{
ON THE LÉVY CONSTANTS FOR QUADRATIC IRRATIONALS
}

\author{
JUN WU \\ (Communicated by David E. Rohrlich)
}

\begin{abstract}
We prove that the set of Lévy constants for quadratic irrationals is dense in $\left[\log \frac{\sqrt{5}+1}{2}, \infty\right)$.
\end{abstract}

\section{INTRODUCTION}

Let $x$ be an irrational number and let $\left[a_{0}(x) ; a_{1}(x), a_{2}(x), \cdots\right]$ be its regular continued fraction expansion. For any $n \geq 1$, we denote by $p_{n}(x) / q_{n}(x):=\left[a_{0}(x)\right.$; $\left.a_{1}(x), a_{2}(x), \cdots, a_{n}(x)\right]$ the $n$th convergent of $x$.

With the conventions $p_{-1}(x)=1, q_{-1}(x)=0, p_{0}(x)=a_{0}(x), q_{0}(x)=1$, we have

$$
\begin{aligned}
& p_{n+1}(x)=a_{n+1}(x) \cdot p_{n}(x)+p_{n-1}(x), \quad n \geq 0, \\
& q_{n+1}(x)=a_{n+1}(x) \cdot q_{n}(x)+q_{n-1}(x), \quad n \geq 0 .
\end{aligned}
$$

The famous Theorem of P. Lévy [6] states that

$$
\lim _{n \rightarrow \infty} \frac{\log q_{n}(x)}{n}=\frac{\pi^{2}}{12 \log 2}
$$

for almost all $x \in \mathbb{R}$ in the sense of Lebesgue.

Definition 1.1. For any irrational number $x \in \mathbb{R}$, if $\lim _{n \rightarrow \infty} \frac{\log q_{n}(x)}{n}$ exists, we say $x$ has a Lévy constant and denote the limit by $\beta(x)$.

Notice that if $x$ has a Lévy constant, then $\beta(x) \geq \log \frac{\sqrt{5}+1}{2}$.

H. Jager and P. Liardet 4] (see also C. Faivre [2]) proved that every quadratic irrational has a Lévy constant. Let

$$
\mathbf{B}=\{\beta(x): x \text { is a quadratic irrational }\} .
$$

E. P. Golubeva [3] showed that $\frac{\pi^{2}}{12 \log 2}$ is a limit point of $\mathbf{B}$; she also presented some evidence that $\log \frac{\sqrt{5}+1}{2}$ may be an isolated point of $\mathbf{B}$. However, we prove that:

Theorem 1.2. B is dense in $\left[\log \frac{\sqrt{5}+1}{2}, \infty\right)$.

Received by the editors January 28, 2005.

2000 Mathematics Subject Classification. Primary 11K50, 11J70.

Key words and phrases. Lévy constant, quadratic irrational.

The author was supported in part by the Kua-Shi-Ji Foundation of Educational Committee and NSFC (10571138). This work was done during the author's visit to the LAMFA, CNRS UMR 6140, Amiens; he would like to thank the institution for their warm hospitality.

(C)2005 American Mathematical Society Reverts to public domain 28 years from publication 
Remark 1.3. If $x=\left[a_{0} ; a_{1}, a_{2}, \cdots, \overline{a_{r+1}, a_{r+2}, \cdots, a_{r+s}}\right]$, from [4] and [1], we have

$$
\lim _{n \rightarrow \infty} \frac{1}{n} \log q_{n}(x)=\frac{1}{s} \log \rho(W)
$$

where

$$
W=\left(\begin{array}{cc}
a_{r+1} & 1 \\
1 & 0
\end{array}\right)\left(\begin{array}{cc}
a_{r+2} & 1 \\
1 & 0
\end{array}\right) \cdots\left(\begin{array}{cc}
a_{r+s} & 1 \\
1 & 0
\end{array}\right)
$$

and $\rho(\cdot)$ denotes the spectral radius of the matrix. Using (1.3), it may be shown that Theorem 1.2 holds, but this nice theorem has not been acknowledged before and the method we used does give a short, simple and self-contained proof of Theorem 1.2 .

\section{Proof of Theorem 1.2}

In this section, we give the proof of Theorem 1.2,

For any $n \geq 1$ and $\left(a_{1}, a_{2}, \cdots, a_{n}\right) \in \mathbb{N}^{n}$, let $q_{n}\left(a_{1}, a_{2}, \cdots, a_{n}\right)$ be the denominator of the finite continued fraction $\left[0 ; a_{1}, a_{2}, \cdots, a_{n}\right]$. The following lemma is proved in [7; we present it here for completeness.

Lemma 2.1. For any $n \geq 1$ and $1 \leq k \leq n$, we have

$$
\frac{a_{k}+1}{2} \leq \frac{q_{n}\left(a_{1}, a_{2}, \cdots, a_{n}\right)}{q_{n-1}\left(a_{1}, \cdots, a_{k-1}, a_{k+1}, \cdots, a_{n}\right)} \leq a_{k}+1\left(q_{0}:=1\right) .
$$

Proof. By (1.2),

$$
\begin{aligned}
& \frac{q_{k}\left(a_{1}, \cdots, a_{k}\right)}{q_{k-1}\left(a_{1}, \cdots, a_{k-1}\right)}=\frac{a_{k} q_{k-1}\left(a_{1}, \cdots, a_{k-1}\right)+q_{k-2}\left(a_{1}, \cdots, a_{k-2}\right)}{q_{k-1}\left(a_{1}, \cdots, a_{k-1}\right)} \leq a_{k}+1, \\
& \frac{q_{k}\left(a_{1}, \cdots, a_{k}\right)}{q_{k-1}\left(a_{1}, \cdots, a_{k-1}\right)} \geq a_{k} \geq \frac{a_{k}+1}{2}, \\
& \frac{q_{k+1}\left(a_{1}, \cdots, a_{k+1}\right)}{q_{k}\left(a_{1}, \cdots, a_{k-1}, a_{k+1}\right)}=\frac{a_{k+1} q_{k}\left(a_{1}, \cdots, a_{k}\right)+q_{k-1}\left(a_{1}, \cdots, a_{k-1}\right)}{a_{k+1} q_{k-1}\left(a_{1}, \cdots, a_{k-1}\right)+q_{k-2}\left(a_{1}, \cdots, a_{k-2}\right)} \\
& =\frac{\left(a_{k+1} a_{k}+1\right) q_{k-1}\left(a_{1}, \cdots, a_{k-1}\right)+a_{k+1} q_{k-2}\left(a_{1}, \cdots, a_{k-2}\right)}{a_{k+1} q_{k-1}\left(a_{1}, \cdots, a_{k-1}\right)+q_{k-2}\left(a_{1}, \cdots, a_{k-2}\right)} \\
& \leq \frac{a_{k+1} a_{k} q_{k-1}\left(a_{1}, \cdots, a_{k-1}\right)}{a_{k+1} q_{k-1}\left(a_{1}, \cdots, a_{k-1}\right)} \\
& \quad+\frac{q_{k-1}\left(a_{1}, \cdots, a_{k-1}\right)+a_{k+1} q_{k-2}\left(a_{1}, \cdots, a_{k-2}\right)}{a_{k+1} q_{k-1}\left(a_{1}, \cdots, a_{k-1}\right)+q_{k-2}\left(a_{1}, \cdots, a_{k-2}\right)} \\
& \leq a_{k}+1 . \\
& \frac{q_{k+1}\left(a_{1}, \cdots, a_{k+1}\right)}{q_{k}\left(a_{1}, \cdots, a_{k-1}, a_{k+1}\right)} \\
& =\frac{\left(a_{k+1} a_{k}+1\right) q_{k-1}\left(a_{1}, \cdots, a_{k-1}\right)+a_{k+1} q_{k-2}\left(a_{1}, \cdots, a_{k-2}\right)}{a_{k+1} q_{k-1}\left(a_{1}, \cdots, a_{k-1}\right)+q_{k-2}\left(a_{1}, \cdots, a_{k-2}\right)} \\
& \geq \\
& =\frac{\left(a_{k+1} a_{k}+1\right) q_{k-1}\left(a_{1}, \cdots, a_{k-1}\right)}{\left(a_{k+1}+1\right) q_{k-1}\left(a_{1}, \cdots, a_{k-1}\right)} \\
& =\frac{a_{k+1} a_{k}+1}{a_{k+1}+1} \geq \frac{a_{k}+1}{2} .
\end{aligned}
$$

Using (1.2) and induction, we get the desired result. 
The following is the proof of Theorem 1.2

Proof. For any $\log \frac{\sqrt{5}+1}{2}<\lambda<\infty$ and any $0<\epsilon<\lambda-\log \frac{\sqrt{5}+1}{2}$, choose $N \in \mathbb{N}$ large enough such that

$$
e^{N\left(\lambda+\epsilon-\log \frac{\sqrt{5}+1}{2}\right)+\log \frac{\sqrt{5}+1}{2}}-1 \geq 2 \cdot e^{N\left(\lambda-\epsilon-\log \frac{\sqrt{5}+1}{2}\right)+\log \frac{\sqrt{5}+1}{2}} .
$$

Choose $b \in \mathbb{N}$ such that

$$
2 \cdot e^{N\left(\lambda-\epsilon-\log \frac{\sqrt{5}+1}{2}\right)+\log \frac{\sqrt{5}+1}{2}}-1 \leq b \leq e^{N\left(\lambda+\epsilon-\log \frac{\sqrt{5}+1}{2}\right)+\log \frac{\sqrt{5}+1}{2}}-1 .
$$

Let $x \in(0,1)$ such that $a_{n}(x)=b$ if $n=k N$ for some $k \in \mathbb{N}$, and $a_{n}(x)=1$ otherwise. $x$ has a purely periodic continued fraction expansion, thus by Lagrange's theorem (see [5], page 56), $x$ is a quadratic irrational.

For any $n \geq N$, there exists $k \in \mathbb{N}$ such that $k N \leq n<(k+1) N$. By Lemma 2.1.

$$
\begin{aligned}
& q_{n}(x) \leq q_{n-k}(1,1, \cdots, 1)(b+1)^{k} \leq c_{1}\left(\frac{\sqrt{5}+1}{2}\right)^{n-k}(b+1)^{k}, \\
& q_{n}(x) \geq q_{n-k}(1,1, \cdots, 1)\left(\frac{b+1}{2}\right)^{k} \geq c_{2}\left(\frac{\sqrt{5}+1}{2}\right)^{n-k}\left(\frac{b+1}{2}\right)^{k},
\end{aligned}
$$

where $c_{1}, c_{2}$ in (2.2) and (2.3) are positive constants which do not depend on $n$. Thus by (2.1), we have

$$
\begin{aligned}
\beta(x) & \leq \limsup _{n \rightarrow \infty} \frac{\log \left(c_{1}\left(\frac{\sqrt{5}+1}{2}\right)^{n-k}(b+1)^{k}\right)}{n} \\
& =\limsup _{n \rightarrow \infty} \frac{k\left(\log (b+1)-\log \frac{\sqrt{5}+1}{2}\right)}{n}+\log \frac{\sqrt{5}+1}{2} \\
& \leq \frac{1}{N}\left(\log (b+1)-\log \frac{\sqrt{5}+1}{2}\right)+\log \frac{\sqrt{5}+1}{2} \leq \lambda+\epsilon, \\
\beta(x) & \geq \liminf _{n \rightarrow \infty} \frac{\log \left(c_{2}\left(\frac{\sqrt{5}+1}{2}\right)^{n-k}\left(\frac{b+1}{2}\right)^{k}\right)}{n} \\
& =\liminf _{n \rightarrow \infty} \frac{k\left(\log (b+1)-\log \frac{\sqrt{5}+1}{2}\right)}{n}+\log \frac{\sqrt{5}+1}{2} \\
& \geq \liminf _{k \rightarrow \infty} \frac{k \cdot\left(\log \frac{b+1}{2}-\log \frac{\sqrt{5}+1}{2}\right)}{(k+1) N}+\log \frac{\sqrt{5}+1}{2} \\
& =\frac{1}{N}\left(\log \frac{b+1}{2}-\log \frac{\sqrt{5}+1}{2}\right)+\log \frac{\sqrt{5}+1}{2} \geq \lambda-\epsilon .
\end{aligned}
$$

Therefore

$$
\beta(x) \in[\lambda-\epsilon, \lambda+\epsilon]
$$




\section{ACKNOWLEDGMENT}

The author is grateful to C. Baxa and C. Faivre for sending him the papers [1] and $[2$.

\section{REFERENCES}

1. C. Baxa, Extremal values of continuants and transcendence of certain continued fractions, Adv. in Appl. Math., 32 (2004), no. 4, 754-790. MR2053844 (2005f:11141)

2. C. Faivre, Distribution of Lévy constants for quadratic numbers, Acta Arith., 61 (1992), no. 1, 13-34. MR1153919 (93c:11057)

3. E. P. Golubeva, The spectrum of Lévy constants for quadratic irrationalities, Zap. Nauchn. Sem. S.-Peterburg. Otdel. Mat. Inst. Steklov. (POMI) 263 (2000), Anal. Teor. Chisel i Teor. Funkts. 16, 20-33, 237, translation in J. Math. Sci. (New York) 110 (2002), no. 6, 3040-3047. MR.1756334 (2001b:11065)

4. H. Jager and P. Liardet, Distributions arithmétiques des dénominateurs des convergents de fraction continues, Indag. Math., 50 (1988), 181-197. MR0952514 (89i:11085)

5. A. Ya. Khintchine, Continued Fractions, P. Noordhoff, Groningen, The Netherlands, 1963. MR 0161834 (28:5038)

6. P. Lévy, Sur les lois de probabilité dont dépendent les quotients complets et incomplets d'une fraction continue, Bull. Soc. Math., 57 (1929), 178-194.

7. J. Wu, A remark on the growth of the denominators of convergents, preprint.

Department of Mathematics, Huazhong University of Science and Technology, Wuhan, Hubei, 430074, People's Republic of China

E-mail address: wujunyu@public.wh.hb.cn 